OPEN ACCESS

Edited by:

Stephanie R. Rainey-Smith,

Murdoch University, Australia

Reviewed by:

Csaba Jozsef Nyakas, Semme/weis University, Hungary

Richard Camicioli,

University of Alberta, Canada

*Correspondence:

Sylvain Moreno

sylvain_moreno@sfu.ca

Received: 12 April 2021

Accepted: 05 July 2021

Published: 16 August 2021

Citation:

Arab A, Christie GJ, Mansouri M, Ahmadzadeh M, Sixsmith A, Ester M and Moreno S (2021)

Moderate-Intensity Physical Activity, Music and Art Activities Preserved Cognitive Health in Older Adults: An

Argument for Social Prescribing Solution.

Front. Aging Neurosci. 13:693791.

doi: 10.3389/fnagi.2021.693791

\section{Moderate-Intensity Physical Activity, Music and Art Activities Preserved Cognitive Health in Older Adults: An Argument for Social Prescribing Solution}

\author{
Ali Arab ${ }^{1}$, Gregory J. Christie ${ }^{2,3}$, Mehrdad Mansouri ${ }^{1}$, Maryam Ahmadzadeh ${ }^{2}$, \\ Andrew Sixsmith ${ }^{4}$, Martin Ester ${ }^{1}$ and Sylvain Moreno ${ }^{2,3 *}$ \\ ${ }^{1}$ School of Computing Science, Simon Fraser University, Burnaby, BC, Canada, ${ }^{2}$ School of Interactive Arts and Technology, \\ Simon Fraser University, Burnaby, BC, Canada, ${ }^{3}$ Science and Technology for Aging Research Institute, Simon Fraser \\ University, Burnaby, BC, Canada, ${ }^{4}$ Department of Gerontology, Simon Fraser University, Vancouver, BC, Canada
}

Introduction: Rates of dementia are projected to increase over the coming years as global populations age. Without a treatment to slow the progression of dementia, many health policies are focusing on preventing dementia by slowing the rate of cognitive decline with age. However, it is unclear which lifestyle changes in old age meaningfully reduce the rate of cognitive decline associated with aging.

Objectives: Use existing, multi-year longitudinal health data to determine if engagement in a variety of different lifestyle activities can slow the rate of cognitive decline as older adults age.

Method: Data from the English Longitudinal Study of Aging was analyzed using a quasi-experimental, efficient matched-pair design inspired by the clinical trial methodology. Changes in short-term memory scores were assessed over a multi-year interval for groups who undertook one of 11 different lifestyle activities, compared to control groups matched across confounding socioeconomic and lifestyle factors.

Results: Two factors, moderate-intensity physical activity and learning activities, resulted in significant positive impact on cognitive function.

Conclusion: Our analysis brings cognitive benefit arguments in favor of two lifestyle activities, moderate-intensity physical activity and learning activities, while rejecting other factors advanced by the literature such as vigorous-intensity physical activity. Those findings justify and encourage the development of new lifestyle health programs by health authorities and bring forward the new health system solution, social prescribing.

Keywords: cognitive function, dementia, Alzheimer's disease, modifiable lifestyle factors, longitudinal observational studies 


\section{INTRODUCTION}

The number of older adults over the age of 65 is increasing globally, which is resulting in a range of challenges for healthcare systems and providers (Lunenfeld, 2008; Christensen et al., 2009). Rates of dementia-a disease that primarily affects older adultsare projected to increase from about 40 million people currently to 125 million by 2050 . No cure exists for dementia and patients require increasing levels of labor-intense care as their symptoms progress, making dementia by far the most expensive end-of-life disease to treat (Kelley et al., 2015). Fortunately, even relatively modest delays in the onset and progression of dementia can result in significant global reductions in this disease; one estimate is that the incidence of $\mathrm{AD}$ would be reduced by approximately $8.6 \%$, or 9.2 million cases if the onset of the disease was delayed by just 1 year (Brookmeyer et al., 2007).

Current pharmacological treatments are ineffective at delaying the onset of various dementias (Birks, 2006; McShane et al., 2019). Most medical recommendations, therefore, focus on preventative health measures (i.e., Social Prescribing; Fancourt et al., 2020), including physical activity, proper diet, and social and cognitively stimulating activities, to maintain cognitive health (Lang et al., 2008; De Oliveira et al., 2014; Norton et al., 2014; Deckers et al., 2015). Although these recommendations are good for maintaining overall physical health, the scientific evidence for maintaining cognitive health is unclear (Christie et al., 2017). Supporting evidence comes largely from observational studies, which often cannot determine whether engagement in a particular activity (e.g., physical exercise, music) has a causal benefit on cognitive health or only a correlational association. Data from randomized control trials have established causal effect between several activities and aging brain health such as physical activity (Cheng, 2016; Ishimaru et al., 2020), meditation and music (Alain et al., 2019). However, these studies are difficult to run and can have their methodological limitations (e.g., poor cross-sectional representation) (Harada et al., 2017).

Here, we investigated a large longitudinal, cross-sectional health dataset, the English Longitudinal Study of Aging, proposing new analytical methods that minimize the effect of confounding variables. Our analysis allowed us to determine if causal inference deems engagement in certain lifestyle activities to have benefit on cognitive health as people age. Based on previous finding (Christie et al., 2017), we hypothesized that engaging in physical activity and learning activities (e.g., art and music classes) would lead to improved cognitive health outcomes.

\section{METHOD}

\subsection{Participants and Sample Data}

We used data from the English Longitudinal Study of Ageing (ELSA), a longitudinal study of community-dwelling people aged 50 and older in England (Steptoe et al., 2013). Participants recruited using multistage-stratified probability sampling. The cohort was first assessed in 2002/2003, and was re-examined in eight examinations (waves), every
2 years. The examination dates are 2004/2005 (wave 2), 2006/2007 (wave 3), 2008/2009 (wave 4), 2010/2011 (wave 5), 2012/2013 (wave 6), 2014/2015 (wave 7), 2016/2017 (wave 8), and 2018/2019 (wave 9). For the purpose of this study, we drew 4,091 participants from waves 1-7 that did not have a missing value in their key variables. Figure 1 shows the age distribution of samples at baseline, wave 1. The statistics for other demographics, social engagement, physical activity, and cognitive function measures are reported in Table 1. Further information on the instrumentation, sampling, recruitment, and data collection procedures can be found at the ELSA's project website https://www.elsa-project.ac.uk/study-documentation.

The outcome variable to assess changes in cognitive function over time was memory index (MI), a computed variable within ELSA comprising three memory subtests on immediate, delayed, and prospective memory. MI ranges from 0 to 27, with 0 zero representing the lowest performance.

Candidate variables were selected a priori to explore causal relation with the outcome variable. Candidate variables included mild/moderate/vigorous physical activity, group membership, tobacco use, contact frequency, friendship quality, and number of close friends. Mild/moderate/vigorous physical activity variables were measured using a 4-point scale (range "more than once a week" to "hardly ever"). Examples of mild activities included laundry and home repairs; moderate intensity activity included gardening, cleaning the car, walking at moderate pace, dancing, and stretching exercises; vigorous intensity included running/jogging, swimming, cycling, aerobics/gym workout, tennis, and digging with a spade. Group membership variables asked the participants to indicate if they are a member of any sports club, social club, church, and if they participate in any learning activities. In particular, learning activities refer to participation in any education, art, or music groups, or evening classes. The values for these measures are 0 and 1 corresponding to No and Yes. Tobacco use was assessed by asking the respondent how many cigarettes they smoke per weekday. To measure contact frequency, respondents were asked how often they meet up with their friends, using a 6-point scale (range "Three or more times a week" to "Less than once a year or never"). Friendship quality captures the perceived social support by the respondents; they were asked how much they think their friends understand their feelings, using a 4-point scale (range "a lot" to "not at all"). As another measure of social support, respondents were asked how many of their friends they would say they have a close relationship with.

Confounding variables are mediator variables that affect both a given candidate variable (which is suspected to be causal) and the outcome variable. Failing to control for confounding variables may result in a misleading correlation, i.e., a possibly strong correlation that is not due to some causal relationships, damaging the validity of the analysis (Spirtes et al., 2000). The confounding variables controlled for in this study include general health and demographics. General health measures the overall health of the participants; The respondents were asked how their health is in general, using a 5-point scale (range "very good" to "very bad"). The demographics used in this study are age, gender, and 




FIGURE 1 | Histogram of the age variable for samples at wave 1

socioeconomic status. Socioeconomic status is a derived variable computed based on other collected economic variables which indicate the decile of the net total wealth.

\subsection{Data Analyses}

We employed a Quasi-experimental design (QED) based on matched pair design to study the effect of candidate variables on MI. In this design, samples are paired randomly such that one sample has been exposed to a potential cause $X_{i}$, known as the treatment, and the other sample has not been exposed to it, while both samples are constrained to be similar with respect to the other potential causes and confounders (Kuhn, 1955; Miettinen, 1968; Mansouri et al., 2018). These constraints guarantee that the effect of the other observed variables on the outcome is controlled, and the random pairing minimizes the effect of latent (unobserved) variables on the outcome.

Figure 2 provides an overview of the analysis method. (i)The method iterates over all candidate variables, for each wave. (ii) Experimental Design: The treatment and control groups were defined based on the value of the candidate variable for the current and the two previous waves. (iii) Cost Matrix Calculation: A cost matrix was calculated, specifying the similarity between each pair of samples from the treatment and control groups. (iv) Matching: A set of matchings were established such that the total dissimilarity cost between matched pairs was minimized while constraining the base memory index to be equal for each pair. If for a treatment sample, two or more control samples have the same minimum distance, one was chosen randomly. (v) Sets of pairs obtained from each wave were merged, and a statistical test was performed to determine the significance of the mean difference of the outcome variable between the treatment and the control cases of the matched pairs. (vi) Finally, after computing the $p$-values for each variable and each wave, all computed $p$ values were adjusted for multiple hypothesis tests (Benjamini and Hochberg, 1995). Details of the method are provided in the following subsections.

\subsection{Preprocessing}

In order to prepare the data for the matched pairs design, a series of pre-processing operations was performed. Variables were normalized to their standard score, with the mean and standard deviation estimated across all samples and all waves. A binary version of the candidate variables $X$ was generated in order to efficiently distinguish between the treatment and control cases. For continuous variables, namely the number of close friends and the number of cigarettes smoked per weekday, based on domain knowledge and variable nature, two thresholds were selected; all values above the higher threshold were converted to one and all values below the lower threshold were converted to zero. For the discrete variables, only one threshold was used for binarization.

\subsection{Identifying Treatment and Control Groups}

To capture the change in cognitive health over time, we consider the difference in MI from one wave to the next one as the outcome. Hence, the treatment group for a candidate variable in a particular wave is defined as samples where the candidate variable is zero at the initial wave and one for the two consecutive waves. Similarly, the control group consists of samples where the 
TABLE 1 | Descriptive statistics for demographics, candidate treatment variables, and the cognitive function measure used in the analysis.

\begin{tabular}{|c|c|c|c|c|c|c|c|c|c|}
\hline & Range & & w1 & W2 & W3 & W4 & W5 & W6 & W7 \\
\hline Sex & $58 \%$ female & & & & & & & & \\
\hline \multirow[t]{2}{*}{ Age } & 50-99 & Mean & 60.31 & & & & & & \\
\hline & & Std & 8.35 & & & & & & \\
\hline \multirow[t]{2}{*}{ Socioeconomic status } & $1-10$ & Mean & 6.32 & 6.24 & 6.2 & 6.03 & 5.94 & 6.04 & 5.96 \\
\hline & & Std & 2.73 & 2.77 & 2.77 & 2.77 & 2.78 & 2.71 & 2.71 \\
\hline \multirow[t]{2}{*}{ Physical health } & $1-5$ & Mean & 2.53 & 2.57 & 2.02 & 2.68 & 2.72 & 2.82 & 2.87 \\
\hline & & Std & 1.08 & 1.05 & 0.82 & 1.04 & 1.05 & 1.06 & 1.06 \\
\hline \multirow[t]{2}{*}{ Mild physical activity } & $1-4$ & Mean & 1.39 & 1.28 & 1.32 & 1.35 & 1.38 & 1.36 & 1.43 \\
\hline & & Std & 0.86 & 0.72 & 0.79 & 0.83 & 0.86 & 0.86 & 0.93 \\
\hline \multirow[t]{2}{*}{ Moderate physical activity } & $1-4$ & Mean & 1.62 & 1.59 & 1.6 & 1.68 & 1.76 & 1.8 & 1.88 \\
\hline & & Std & 1 & 0.98 & 1.01 & 1.07 & 1.12 & 1.16 & 1.22 \\
\hline \multirow[t]{2}{*}{ Vigorous physical activity } & $1-4$ & Mean & 2.93 & 2.95 & 3.01 & 3.03 & 3.1 & 3.13 & 3.21 \\
\hline & & Std & 1.26 & 1.25 & 1.25 & 1.24 & 1.21 & 1.21 & 1.18 \\
\hline \multirow[t]{2}{*}{ Friendship Quality } & $1-4$ & Mean & 1.87 & 1.88 & 1.83 & 1.86 & 1.88 & 1.87 & 1.8 \\
\hline & & Std & 0.78 & 0.76 & 0.75 & 0.76 & 0.76 & 0.75 & 0.73 \\
\hline \multirow[t]{2}{*}{ Number of close friends } & $0-98$ & Mean & 3.22 & 3.88 & 4.43 & 3.65 & 3.67 & 3.61 & 3.74 \\
\hline & & Std & 3.66 & 4.45 & 6.6 & 2.97 & 3.16 & 2.96 & 3.44 \\
\hline \multirow[t]{2}{*}{ Contact frequency } & $1-6$ & Mean & 2.46 & 2.5 & 2.48 & 2.49 & 2.49 & 2.51 & 3.73 \\
\hline & & Std & 1.02 & 1.04 & 1.03 & 1.07 & 1.08 & 1.09 & 0.56 \\
\hline \multirow[t]{2}{*}{ Sport club membership } & $0-1$ & Mean & 0.26 & 0.25 & 0.25 & 0.25 & 0.24 & 0.25 & 0.26 \\
\hline & & Std & 0.44 & 0.44 & 0.43 & 0.44 & 0.43 & 0.43 & 0.44 \\
\hline \multirow[t]{2}{*}{ Church membership } & $0-1$ & Mean & 0.23 & 0.24 & 0.23 & 0.24 & 0.23 & 0.24 & 0.24 \\
\hline & & Std & 0.42 & 0.43 & 0.42 & 0.43 & 0.42 & 0.42 & 0.43 \\
\hline \multirow[t]{2}{*}{ Social club membership } & $0-1$ & Mean & 0.2 & 0.2 & 0.19 & 0.2 & 0.2 & 0.19 & 0.19 \\
\hline & & Std & 0.4 & 0.4 & 0.39 & 0.4 & 0.4 & 0.39 & 0.39 \\
\hline \multirow[t]{2}{*}{ Learning activities } & $0-1$ & Mean & 0.18 & 0.16 & 0.15 & 0.14 & 0.15 & 0.15 & 0.15 \\
\hline & & Std & 0.39 & 0.37 & 0.36 & 0.35 & 0.36 & 0.36 & 0.36 \\
\hline \multirow[t]{2}{*}{ Smoking } & $0-60$ & Mean & 1.78 & 1.49 & 1.3 & 1.17 & 1.05 & 0.92 & 0.78 \\
\hline & & Std & 5.67 & 5.2 & 4.78 & 4.66 & 4.31 & 4.04 & 3.72 \\
\hline \multirow[t]{2}{*}{ Memory index } & $3-24$ & Mean & 14.55 & 14.88 & 14.93 & 14.76 & 14.62 & 14.59 & 13.95 \\
\hline & & Std & 3.17 & 3.21 & 3.34 & 3.38 & 3.54 & 3.65 & 4.04 \\
\hline
\end{tabular}

candidate variable is zero for both the previous wave and the current wave. The reason for defining one treatment and control group per wave is to control the impact of time-dependent latent variables such as age and social scale events.

\subsection{Pairing}

In this step, samples from treatment and control groups are paired together. To control the effect of confounding variables, the features of each pair are constrained to have a minimum distance. A hard constraint was imposed on the base memory index to make them equal for the treatment and control pairs. A sample is represented by a vector consisting of the other potential causes and the confounding variables. The distance between two samples is defined as the Euclidean distance of their corresponding vectors. In cases where multiple pairings with the same distance are possible for a sample, one is randomly selected to add the randomness component to the matched pairs design. Once the distance between all pairs is calculated, Hungarian algorithm (Kuhn, 1955) is applied to get an optimal pairing.
To define the pairing process formally, we introduce the following notation; $\left[Y^{i}\right]^{t}(1 \leq t \leq T, 1 \leq i \leq N)$ is the target value of sample $i$ in wave $t$, where $T$ is the number of time points, and $N$ is the number of samples; $\left[X_{j}^{i}\right]^{t}(1 \leq t \leq T, 1 \leq i \leq N, 1 \leq j \leq M)$, is the $j t h$ candidate variable of sample $i$ in wave $t$, where $M$ is the number of candidate variables; and $\left[Z_{j}^{i}\right]^{t}(1 \leq t \leq T, 1 \leq i \leq N, 1 \leq j \leq K)$, is the $j^{\text {th }}$ confounding variable of sample $i$ in wave $t$, where $K$ is the number of confounding variables.

As the input, the Hungarian algorithm takes the cost matrix specifying the cost of each pairing. Output of the algorithm is a matching where the total cost is minimized. As usually the size of treatment and control groups are different, the number of matched pairs is the minimum size of treatment and control group. The more similar a pair, the lower the cost. The Hungarian algorithm solves the pairing of treatment and control cases as an Assignment problem:

$$
\begin{aligned}
& u^{*}=\operatorname{argmin}_{u} \sum_{n_{t}} \sum_{n_{c}} D\left(i, i^{\prime}, t\right) u_{i i^{\prime}} \\
& \text { such that } u_{i i^{\prime}} \in\{0,1\} \quad \text { and } \quad \sum_{i=1}^{n_{t}} u_{i i^{\prime}}=1 \quad \text { and } \quad \sum_{i^{\prime}=1}^{n_{c}} u_{i i^{\prime}}=1
\end{aligned}
$$



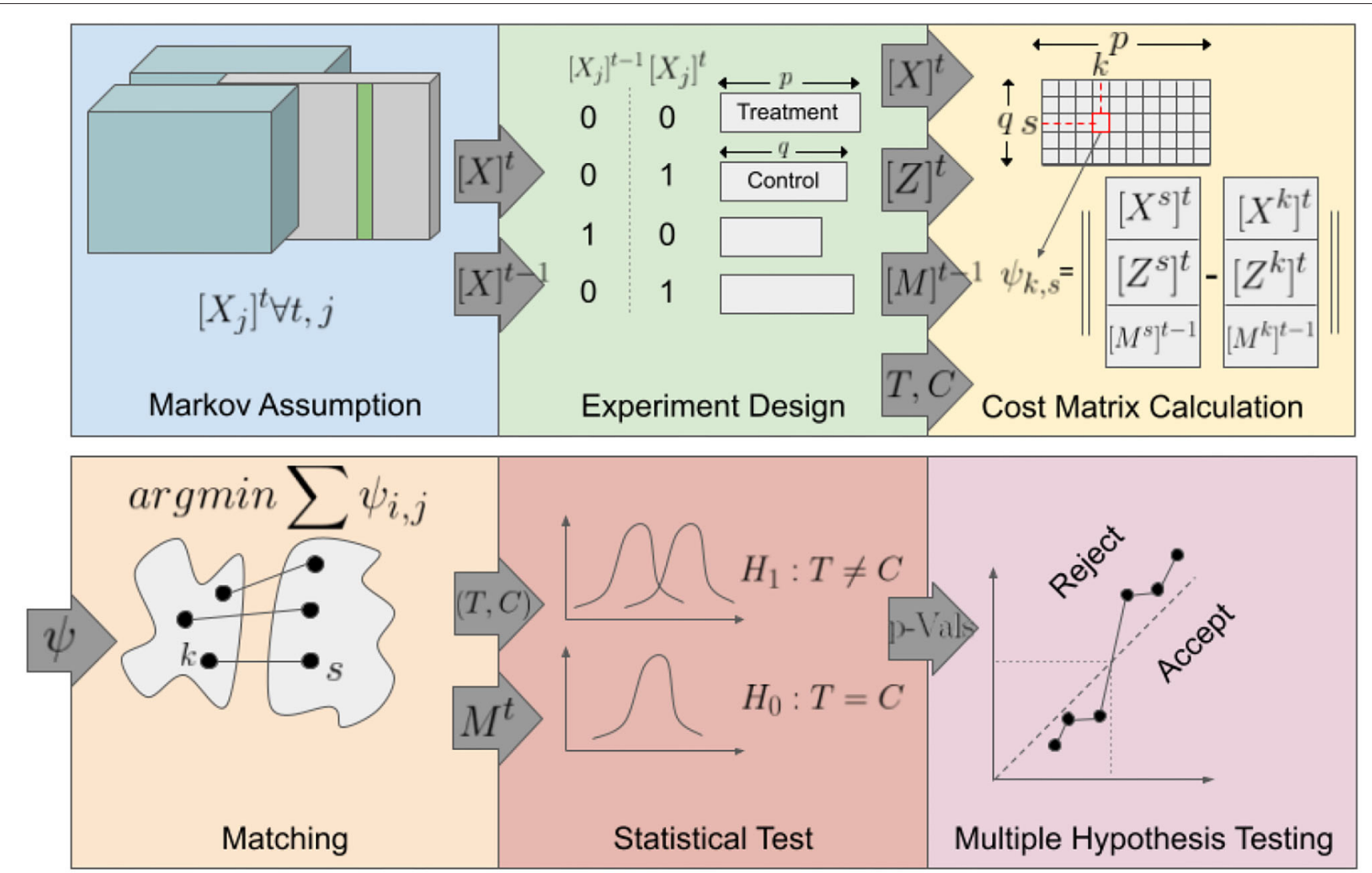

FIGURE 2 | Overview of the method; schematic overview of the steps involved in the QED.

where $u^{*}$ is the optimal assignment, $n_{c}$ is the number of control samples and $n_{t}$ is the number of treatment samples. In this formulation, $u_{i i^{\prime}}=1$ indicates that sample $i$ of treatment set and sample $i^{\prime}$ of control set are paired, and $u_{i i^{\prime}}=0$ indicates otherwise. $D\left(i, i^{\prime}, t\right)$ is the matching distance between treatment sample $i$ and control sample $i^{\prime}$ at time $t$, for variable $v$ is composed of three components:

$$
\begin{aligned}
D\left(i, i^{\prime}, t, v\right)= & D_{\text {confounders }}\left(i, i^{\prime}, t\right)+D_{\text {treatments }}\left(i, i^{\prime}, t, v\right) \\
& +\beta * D_{\text {target }}\left(i, i^{\prime}, t\right)
\end{aligned}
$$

where $\beta$ is the weight given to the target variable during the matching. The reason for allocating a component for each of the confounders, treatment, and target variable is that a high number of variables in one category does not overshadow the variables in other categories. A high weight is used for $D_{\text {target }}$ to make sure that the two matched samples have similar conditions at the baseline in terms of the target variable. The subcomponents are defined as following:

$$
\begin{aligned}
& D_{\text {target }}\left(i, i^{\prime}, t\right)=\left[Y^{i}\right]^{t-2}-\left[Y^{i^{\prime}}\right]^{t-2} \\
& D_{\text {Confounder }}\left(i, i^{\prime}, t\right)=\sum_{j=0}^{K-1}\left(\left[Z_{j}^{i}\right]^{t}-\left[Z_{j}^{i^{\prime}}\right]^{t}\right) \\
& D_{\text {treatment }}\left(i, i^{\prime}, t, v\right)=\sum_{\substack{j=0 \\
j \neq v}}^{M-1}\left(\left[X_{j}^{i}\right]^{t}-\left[X_{j}^{i^{\prime}}\right]^{t}\right)
\end{aligned}
$$

\subsection{Statistical Test}

Considering that the values for the outcome are continuous and skewed, the potential causes are binary, and the samples are paired, the Wilcoxon Signed-Rank test is used to evaluate the impact of a potential cause. The Wilcoxon Signed-Rank test for our QED evaluates the null hypothesis that there is no significant difference in the change of MI because of the value of the candidate variable. This provides us with one $p$-value for each candidate variable and each wave.

\subsection{Adjusting the $\boldsymbol{P}$-Values}

Evaluating the causality of multiple candidate variables for MI produces a large number of hypotheses. This increases the chance of some of these hypotheses being incorrectly accepted by chance, which is known as the multiple comparisons problem. A standard approach to counteract the multiple comparisons problem is to adjust $p$-values and the threshold to accept them based on the false discovery rate. We use the Benjamin-Hochberg adjustment method for this purpose.

The outline of our approach is presented is Algorithm 1. The source code is made publicly available to facilitate the usage and reproducibility of the experimental results ${ }^{1}$.

\footnotetext{
${ }^{1}$ https://github.com/causal-discovery-elsa/causal_discovery_elsa.
} 
TABLE $2 \mid P$-values and sample sizes associated with hypothesis testing across all waves and merged population.

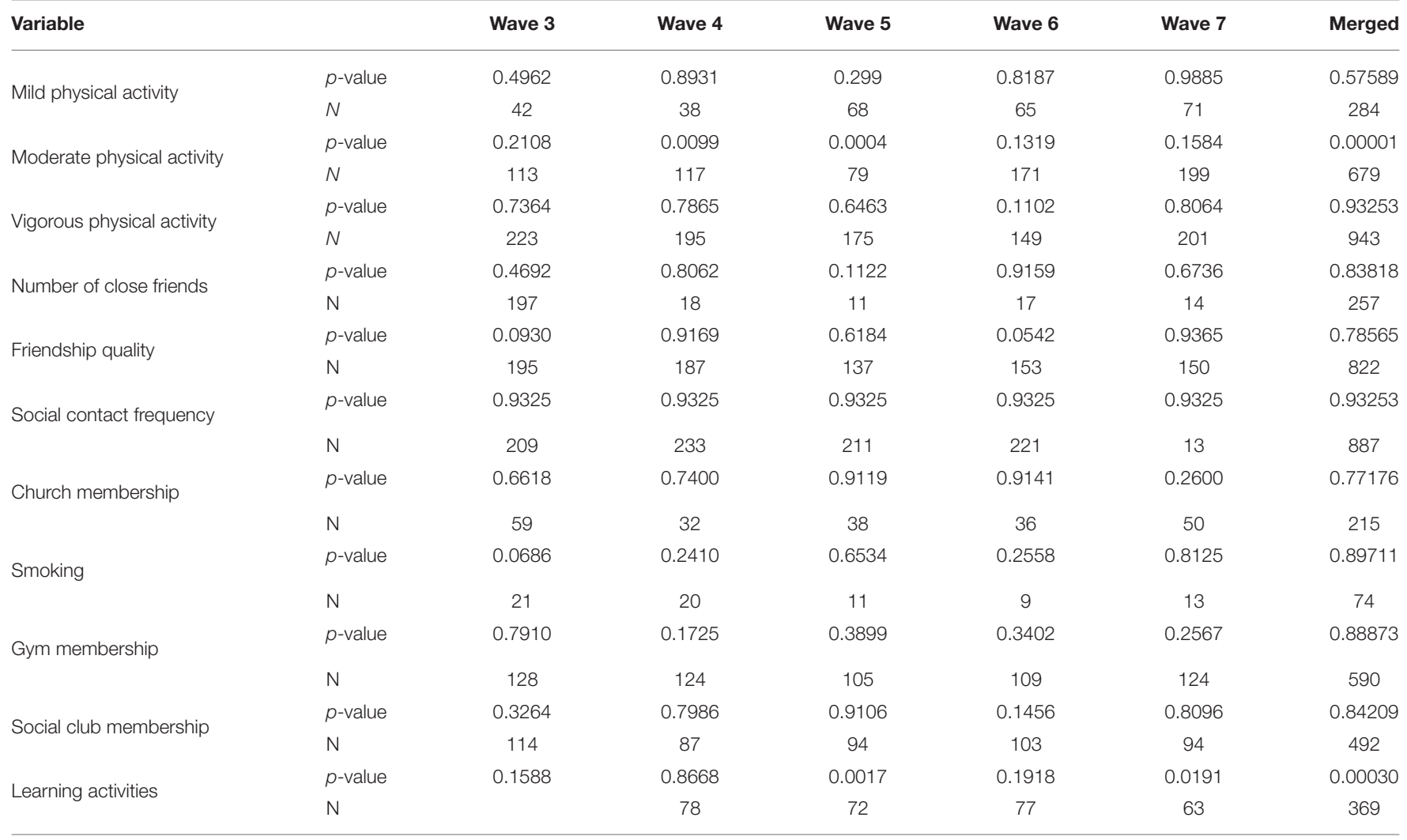

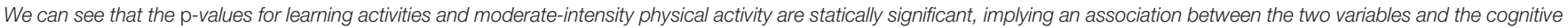
function measure, MI. N, sample size.






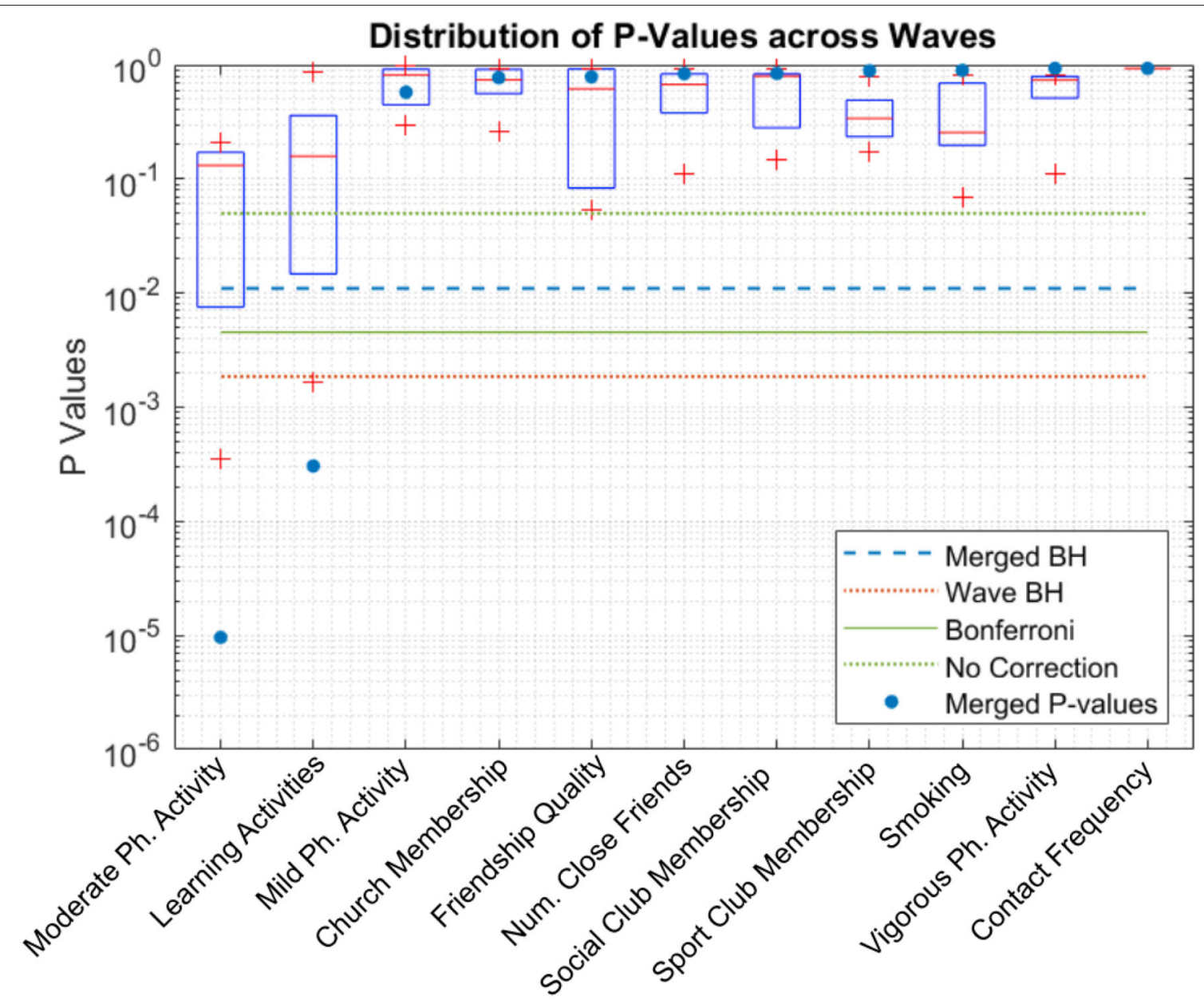

FIGURE 4 | Boxplot of $p$-values of each candidate. Learning activities and moderate-intensity physical activity pass the statistical test after $p$-value adjustment even for the more conservative adjustment method, Bonferroni. "Merged BH" and "Wave BH" indicate the significance thresholds for Benjamini-Hochberg method performed on $p$-values of merged waves and individual waves, respectively.

\section{RESULTS}

The set of $p$-values for each combination of one variable and one wave and also for each variable in the merged population is provided in Table 2. The $p$-value for individual waves was computed based on the treatment and control group identified at each wave, whereas the last column shows the $p$-value for the merged population across all five waves. The sample sizes used to compute the $p$-values are presented below each variable.

Figure 3A shows the curve corresponding to the $p$-values of candidate variables across all waves, with the blue line depicting the Benjamini-Hochberg adjusted threshold. Among the candidate variables, learning activities and moderateintensity physical activity pass multiple hypothesis testing. The distribution of $p$-values is very healthy, with a long linear trail of evenly distributed $p$-values, as would typically be observed from random variables, and ending with few exponentially smaller $p$ values corresponding to statistically significant factors and their covariates (Oyeniran and Chen, 2016).
The corresponding curve for the merged population is shown in Figure 3B. As before, the blue line depicts the BH-adjusted 5\% significance threshold. Consistent with the previous figure, both learning activities and moderate-intensity physical activity pass multiple hypothesis testing.

Figure 4 shows the boxplots of $p$-values for each candidate variable. Horizontal lines depict various thresholds for rejecting the null hypothesis without correction for familywise error, Bonferroni correction (Oyeniran and Chen, 2016) and Benjamini-Hochberg correction. Learning activities and moderate-intensity physical activity remain significant even at the more conservative Bonferroni level.

Finally, to evaluate the magnitude of the improvement expected from each treatment, we measured the mean difference in MI between treatment and control group pairs (Figure 5). Blue and red bars correspond to the merged population analysis and the per-wave analysis, respectively; in the latter case, bar height is the average of observed improvement overall five waves. For the merged population analysis, the two highest bars again 


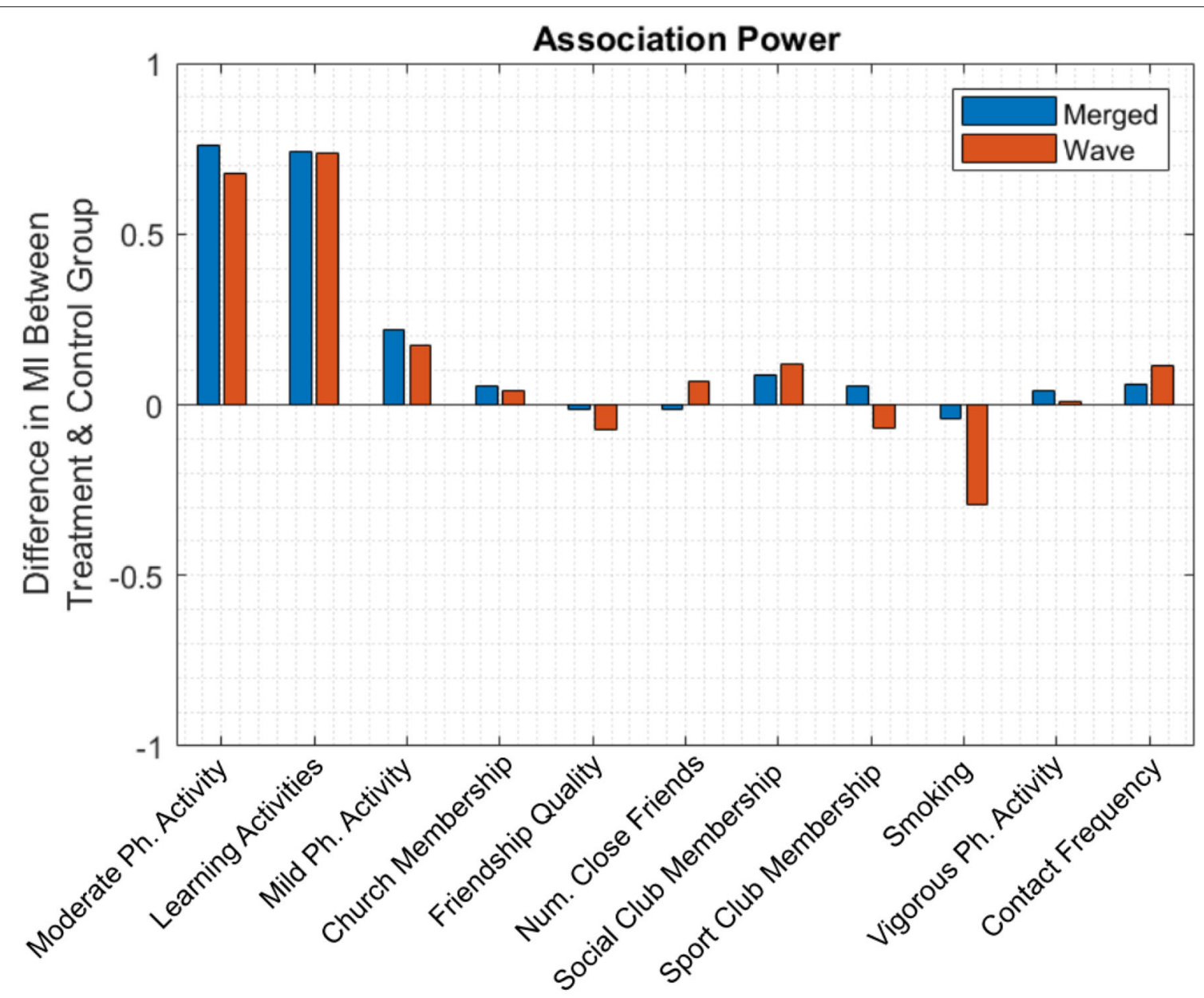

FIGURE 5 | The mean difference in MI between treatment and control group. Engagement in moderate-intensity physical activity and learning activities leads to approximately 0.75 points increase in Ml.

correspond to the two previously identified significant candidate variables. Although significant, the benefit from each of these activities was relatively modest, leading to an improvement in MI of approximately 0.75 points on a 27 -point scale.

In the per-wave analysis, smoking had a relatively large negative value, suggesting that MI decreased as the number of cigarettes smoked increased. However, due to a relative lack of participant data, this comparison lacks statistical power and was not identified as a statistically significant factor.

\section{DISCUSSION}

For many years, researchers have sought to ascertain whether certain lifestyle changes (e.g., physical activity) in later life can preserve cognitive health during aging. Here, our findings settle part of this debate by analyzing a large, longitudinal study of aging containing detailed information on cognitive function and real-world setting engagement in various life activities. To date, many of the existing studies of lifestyle effect on cognitive health have focused on correlational changes in cognition over time (Sona et al., 2012). Inferring causation from such studies is challenging because confounding effects cannot be ruled out. Causal discovery strives to identify cause-effect relations between a set of treatment and outcome variables, where any change in a causal treatment would result in a change in the outcome (Nguyen, 2018). The gold standard method to test causality is with randomized control trials, but RCT studies are notoriously difficult to conduct in the field of longitudinal cognitive health. QED is a competent replacement for randomized controlled trials in the evaluation of causal hypotheses using observational data when the sample size is not a limiting factor. QEDs are designed to deal with the lack of random assignment in observational data by sampling from the population such that the impact of selection bias and confounding is minimized. In this study, we adopted a QED based on matched pair design to investigate the effect of undertaking and maintaining changes in various lifestyle factors and how these factors influenced long-term cognitive health.

The results of these analyses revealed only two lifestyle activities that led to improved long-term cognitive outcomes: moderate-intensity physical activity and engagement in learning activities, such as education, art, music groups, or evening classes. Specifically, older adults who engaged in moderate-intensity 


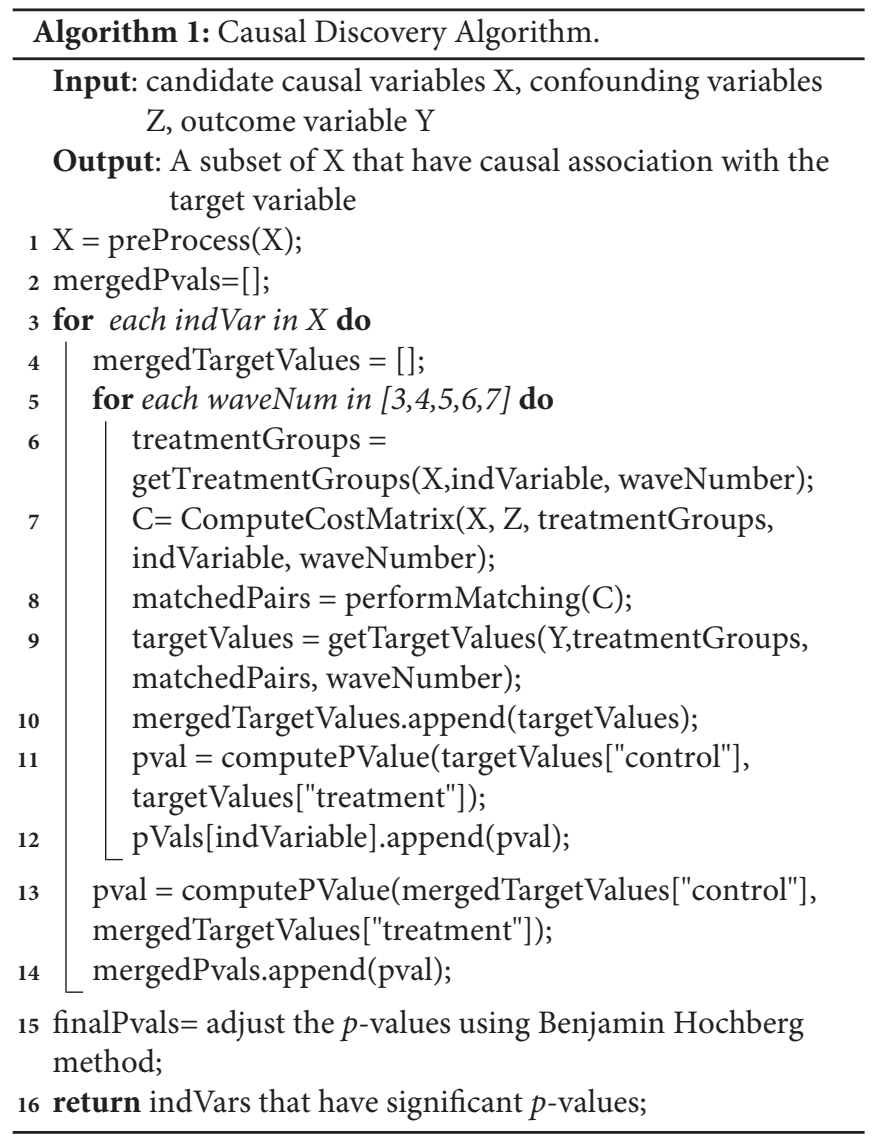

physical activities, such as gardening and walking, for a period of at least 4 years with a frequency of at least three times per week, gained one-third standard deviations from their baseline of memory score. Similarly, older adults who participated in learning activities for at least 4 years experienced a similar level of improvement in their cognitive health.

Broadly, these findings dovetail with past studies that sought to determine how lifestyle activities affect cognitive aging. For example, a recent meta-analysis by Woods et al. (2012) determined that cognitive stimulation improves cognition in individuals with mild cognitive impairment and moderate dementia over and above any benefit from medication. Likewise, studies of same-sex twins found that participation in leisure activities during early and middle adulthood-and participation in intellectual-cultural activities in particularwas associated with a reduced risk of Alzheimer's disease (Crowe et al., 2003).

In the present study, other lifestyle activities previously proposed as beneficial for healthy cognitive aging, such as active social participation and vigorous-intensity physical activity, did not pass hypothesis testing. We do not rule out the possibility that these and other activities may also have a positive effect on cognitive aging, which was not observed in the present study given certain limitations outlined below. Likewise, several additional issues remain to be investigated, including whether cognitive benefits are affected by the vigor or frequency of learning activities; whether exercise and learning activities are tapping into overlapping or separable neurcognitive circuitry; the added cognitive health benefit, if any, of continued engagement across longer time scales; of engagement in combinations of multiple life activities; of the duration of the cognitive protective factor following discontinuation of a given life activity; and understanding how these cognitive benefits change across older adults' lifespans.

A critical piece of converging evidence lies in determining the neuroanatomical effects of lifestyle activities and how these affect brain structure and function over time. Moderate exercise and learning activities likely both recruit wide-scale neuronal involvement for a range of activities that can include movementrelated learning and delicate motor regulation. We consider it plausible that exercise and learning activities induce neuroplastic changes in the brain that counter age-related cognitive decline. Similar neuroplastic explanations have been advanced to account for cognitive improvements in other pathological populations (Särkämö et al., 2014, 2016) and healthy populations (Hötting and Röder, 2013; Moussard et al., 2016; Alain et al., 2019) and may be instantiated through a combination of increased synaptic and dendritic receptors, and changes in neuronal growth factors (Kraft, 2012; Hötting and Röder, 2013; Moreno and Bidelman, 2014); for reviews of the neurobiology of plasticity in aging see Freret et al. (2015) and Gelfo et al. (2018). Future studies will help clarify whether moderate exercise and learning activities are tapping into separable underlying mechanisms or whether these activities (and others) are accessing a unified underlying mechanism that can promote healthy cognitive aging.

As with any longitudinal study, our analysis suffers from a number of limitations. First, the data in this study was selfreported and retrospective, and could therefore be different from participants' actual behaviors. This can be exacerbated when the measurement techniques change over time, leading to a wrong inference of having found a causal relation while a developmental change actually occurring. Second, the study design included only individuals who participated in all seven waves of the ELSA (to mitigate attrition bias) and who engaged in a new lifestyle activity for at least two subsequent waves (to demonstrate a treatment effect). This may limit the extent to which the present findings apply to the broader public and may have increased the incidence of Type-II error. To increase generalizability, further replication studies with a wider variety of data sources will be required.

Nevertheless, the present results represent an important, causal discovery analysis on a large, longitudinal population. They confirm the significance of the relationship between moderate-intensity physical activities, learning activities, and long-term cognitive functioning. They also largely rule out important alternatives (i.e., spurious correlations) such as engaging in greater levels of physical activity and community classes simply because they have greater cognitive health to do so. We encourage additional research in this space and the development of community health guidelines to promote healthy cognitive aging. 


\section{CONCLUSION}

Typical onset of dementia is at approximately age 80, and with the oldest of the baby boom generation approaching this age, there is a narrowing window with which to proactively maintain cognitive health. The results from the present study brings an answer to the baby boom generation's key question, engagement in moderate-intensity physical activity and learning activities may provide modest but significant protection against cognitive decline in aging. Therefore, we recommend the mobilization of this knowledge into community initiatives aimed at older adults and developing health policy encouraging programs such as social prescribing.

\section{DATA AVAILABILITY STATEMENT}

Publicly available datasets were analyzed in this study. This data can be found at: https://www.elsa-project.ac.uk/.

\section{AUTHOR CONTRIBUTIONS}

AA had full access to all of the data in the study and takes responsibility for the integrity of the data and the accuracy of the data analysis. ME and SM: study concept and design. AA, GC, and MM: drafting of the manuscript. AA and MM: statistical analysis. ME, SM, and GC: obtained funding. MA: administrative

\section{REFERENCES}

Alain, C., Moussard, A., Singer, J., Lee, Y., Bidelman, G. M., and Moreno, S. (2019). Music and visual art training modulate brain activity in older adults. Front. Neurosci. 13:182. doi: 10.3389/fnins.2019.00182

Benjamini, Y., and Hochberg, Y. (1995). Controlling the false discovery rate: a practical and powerful approach to multiple testing. J. R. Stat. Soc. Ser. B 57, 289-300. doi: 10.1111/j.2517-6161.1995.tb02031.x

Birks, J. S. (2006). Cholinesterase inhibitors for Alzheimer's disease. Cochr. Datab. Syst. Rev. 1:CD005593. doi: 10.1002/14651858.CD005593

Brookmeyer, R., Johnson, E., Ziegler-Grahamm, K., and Arrighi, H. M. (2007). Forecasting the global prevalence and burden of Alzheimer's disease. Alzheimer's Dement. 3(3S Part 3), S168-S168. doi: 10.1016/j.jalz.2007.04.087

Cheng, S.-T. (2016). Cognitive reserve and the prevention of dementia: the role of physical and cognitive activities. Curr. Psychiatry Rep. 18, 1-12. doi: 10.1007/s11920-016-0721-2

Christensen, K., Doblhammer, G., Rau, R., and Vaupel, J. W. (2009). Ageing populations: the challenges ahead. Lancet 374, 1196-1208. doi: 10.1016/S0140-6736(09)61460-4

Christie, G. J., Hamilton, T., Manor, B. D., Farb, N. A., Farzan, F., Sixsmith, A., et al. (2017). Do lifestyle activities protect against cognitive decline in aging? A review. Front. Aging Neurosci. 9:381. doi: 10.3389/fnagi.2017.00381

Crowe, M., Andel, R., Pedersen, N. L., Johansson, B., and Gatz, M. (2003). Does participation in leisure activities lead to reduced risk of Alzheimer's disease? A prospective study of swedish twins. J. Gerontol. Ser. B Psychol. Sci. Soc. Sci. 58, P249-P255. doi: 10.1093/geronb/58.5.P249

De Oliveira, T. C. G., Soares, F. C., De Macedo, L. D. E. D., Diniz, D. L. W. P., Bento-Torres, N. V. O., and Picanço-Diniz, C. W. (2014). Beneficial effects of multisensory and cognitive stimulation on age-related cognitive decline in long-term-care institutions. Clin. Interv. Aging 9:309. doi: 10.2147/CIA.S54383

Deckers, K., van Boxtel, M. P., Schiepers, O. J., de Vugt, M., Munoz Sanchez, J. L., Anstey, K. J., et al. (2015). Target risk factors for dementia prevention: a systematic review and delphi consensus study on the and technical support. ME and SM: study supervision. All authors acquisition, analysis, or interpretation of data, critical revision of the manuscript for important intellectual content. All authors provided input into the design, edited and revised the manuscript, and read and approved the final manuscript.

\section{FUNDING}

This study was supported by grants from the Simon Fraser University Community Trust Endowment Fund and an AGEWELL Catalyst program (AW-SPC07-2019 and AWCAT-2017107).

\section{ACKNOWLEDGMENTS}

The data were made available through the UK Data Archive (UKDA). The English Longitudinal Study of Ageing was developed by a team of researchers based at the University College London, NatCen Social Research, and the Institute for Fiscal Studies. The data were collected by NatCen Social Research. The funding is currently provided by the National Institute of Aging (R01AG017644), and a consortium of UK government departments coordinated by the National Institute for Health Research. The developers and funders of ELSA and the Archive do not bear any responsibility for the analyses or interpretations presented here.

evidence from observational studies. Int. J. Geriatr. Psychiatry 30, 234-246. doi: 10.1002 /gps.4245

Fancourt, D., Steptoe, A., and Cadar, D. (2020). Community engagement and dementia risk: time-to-event analyses from a national cohort study. $J$. Epidemiol. Commun. Health 74, 71-77. doi: 10.1136/jech-2019-213029

Freret, T., Gaudreau, P., Schumann-Bard, P., Billard, J.-M., and PopaWagner, A. (2015). Mechanisms underlying the neuroprotective effect of brain reserve against late life depression. J. Neural Trans. 122, 55-61. doi: 10.1007/s00702-013-1154-2

Gelfo, F., Mandolesi, L., Serra, L., Sorrentino, G., and Caltagirone, C. (2018). The neuroprotective effects of experience on cognitive functions: evidence from animal studies on the neurobiological bases of brain reserve. Neuroscience 370, 218-235. doi: 10.1016/j.neuroscience.2017.07.065

Harada, C. N., Natelson, M. C., and Triebel, K. L. (2017). Normal cognitive aging. Clin. Geriatr. Med. 29, 737-752. doi: 10.1016/j.cger.2013.07.002

Hötting, K., and Röder, B. (2013). Beneficial effects of physical exercise on neuroplasticity and cognition. Neurosci. Biobehav. Rev. 37, 2243-2257. doi: 10.1016/j.neubiorev.2013.04.005

Ishimaru, D., Tanaka, H., Nagata, Y., Takabatake, S., and Nishikawa, T. (2020). Physical activity in severe dementia is associated with agitation rather than cognitive function. Am. J. Alzheimer's Dis. Other Dement. 35:1533317519871397. doi: 10.1177/1533317519871397

Kelley, A. S., McGarry, K., Gorges, R., and Skinner, J. S. (2015). The burden of health care costs for patients with dementia in the last 5 years of life. Ann. Intern. Med. 163, 729-736. doi: 10.7326/M15-0381

Kraft, E. (2012). Cognitive function, physical activity, and aging: possible biological links and implications for multimodal interventions. Aging Neuropsychol. Cogn. 19, 248-263. doi: 10.1080/13825585.2011.645010

Kuhn, H. W. (1955). The Hungarian method for the assignment problem. Naval Res. Logist. Quar. 2, 83-97. doi: 10.1002/nav.3800020109

Lang, I. A., Llewellyn, D. J., Langa, K. M., Wallace, R. B., Huppert, F. A., and Melzer, D. (2008). Neighborhood deprivation, individual socioeconomic status, and cognitive function in older people: analyses from 
the English longitudinal study of ageing. J. Am. Geriatr. Soc. 56, 191-198. doi: 10.1111/j.1532-5415.2007.01557.x

Lunenfeld, B. (2008). An Aging World-demographics and challenges. Gynecol. Endocrinol. 24, 1-3. doi: 10.1080/09513590701718364

Mansouri, M., Yuan, B., Ross, C. J., Carleton, B. C., and Ester, M. (2018). Hume: large-scale detection of causal genetic factors of adverse drug reactions. Bioinformatics 34, 4274-4283. doi: 10.1093/bioinformatics/bty475

McShane, R., Westby, M. J., Roberts, E., Minakaran, N., Schneider, L., Farrimond, L. E., et al. (2019). Memantine for dementia. Cochr. Datab. Syst. Rev. 3:CD003154. doi: 10.1002/14651858.CD003154.pub6

Miettinen, O. S. (1968). The matched pairs design in the case of all-or-none responses. Biometrics 339-352. doi: 10.2307/2528039

Moreno, S., and Bidelman, G. M. (2014). Examining neural plasticity and cognitive benefit through the unique lens of musical training. Hear. Res. 308, 84-97. doi: 10.1016/j.heares.2013.09.012

Moussard, A., Bermudez, P., Alain, C., Tays, W., and Moreno, S. (2016). Life-long music practice and executive control in older adults: an event-related potential study. Brain Res. 1642, 146-153. doi: 10.1016/j.brainres.2016.03.028

Nguyen, T. K. H. (2018). Structure learning of graphs for count data (Ph.D. thesis). University of Padua, Padua, Italy.

Norton, S., Matthews, F. E., Barnes, D. E., Yaffe, K., and Brayne, C. (2014). Potential for primary prevention of Alzheimer's disease: an analysis of population-based data. Lancet Neurol. 13, 788-794. doi: 10.1016/S1474-4422(14)70136-X

Oyeniran, O., and Chen, H. (2016). Estimating the proportion of true null hypotheses in multiple testing problems. J. Probab. Stat. 2016:3937056. doi: $10.1155 / 2016 / 3937056$

Särkämö, T., Altenmüller, E., Rodríguez-Fornells, A., and Peretz, I. (2016). Music, brain, and rehabilitation: emerging therapeutic applications and potential neural mechanisms. Front. Hum. Neurosci. 10:103. doi: 10.3389/fnhum.2016.00103

Särkämö, T., Tervaniemi, M., Laitinen, S., Numminen, A., Kurki, M., Johnson, J. K., et al. (2014). Cognitive, emotional, and social benefits of regular musical activities in early dementia: randomized controlled study. Gerontologist 54, 634-650. doi: 10.1093/geront/gnt100
Sona, A., Zhang, P., Ames, D., Bush, A. I., Lautenschlager, N. T., Martins, R., et al. (2012). Predictors of rapid cognitive decline in Alzheimer's disease: results from the Australian imaging, biomarkers and lifestyle (AIBL) study of ageing. Int. Psychogeriatr. 24, 197-204. doi: 10.1017/S10416102110 01335

Spirtes, P., Glymour, C. N., Scheines, R., and Heckerman, D. (2000). Causation, Prediction, and Search. Cambridge: MIT Press. doi: 10.7551/mitpress/1754.001.0001

Steptoe, A., Breeze, E., Banks, J., and Nazroo, J. (2013). Cohort profile: the english longitudinal study of ageing. Int. J. Epidemiol. 42, 1640-1648. doi: 10.1093/ije/dys168

Woods, B., Aguirre, E., Spector, A. E., and Orrell, M. (2012). Cognitive stimulation to improve cognitive functioning in people with dementia. Cochr. Database Syst. Rev. 2:CD005562. doi: 10.1002/14651858.CD005562.pub2

Conflict of Interest: The authors declare that the research was conducted in the absence of any commercial or financial relationships that could be construed as a potential conflict of interest.

Publisher's Note: All claims expressed in this article are solely those of the authors and do not necessarily represent those of their affiliated organizations, or those of the publisher, the editors and the reviewers. Any product that may be evaluated in this article, or claim that may be made by its manufacturer, is not guaranteed or endorsed by the publisher.

Copyright (c) 2021 Arab, Christie, Mansouri, Ahmadzadeh, Sixsmith, Ester and Moreno. This is an open-access article distributed under the terms of the Creative Commons Attribution License (CC BY). The use, distribution or reproduction in other forums is permitted, provided the original author(s) and the copyright owner(s) are credited and that the original publication in this journal is cited, in accordance with accepted academic practice. No use, distribution or reproduction is permitted which does not comply with these terms. 